\title{
CFD-based Design of LOX Pump Inlet Duct for Reduced Dynamic Loads
}

\author{
Jeffry Rothermel, ${ }^{\#}$ Daniel J. Dorney, ${ }^{*}$ and Suzanne M. Dorney ${ }^{+}$ \\ NASA Marshall Space Flight Center \\ Applied Fluid Dynamics Analysis Group \\ MSFC, AL 35812
}

\begin{abstract}
Numerical simulations have been completed for a variety of designs for a 90 deg elbow duct. The objective is to identify a design that minimizes the dynamic load entering a LOX turbopump located at the elbow exit. Designs simulated to date indicate that simpler duct geometries result in lower losses. Benchmark simulations have verified that the compressible flow code used in this study is applicable to these incompressible flow simulations.
\end{abstract}

\section{NOTATION}

$\mathrm{C}_{\mathrm{p}} \quad$ - pressure coefficient

d - pipe diameter

m - mass flow $(\mathrm{lbm} / \mathrm{sec})$

P - static pressure (psia)

$P_{\text {ref }} \quad$ - static pressure (psia) one diameter upstream of pipe bend

$\mathrm{R} \quad$ - pipe bend radius

$\operatorname{Re} \quad$ - turbulent Reynolds number

$U$ - streamwise velocity

$\mathrm{U}_{\mathrm{B}} \quad$ - bulk velocity

$\theta \quad$ - bend angle

$\rho$ - density

\section{INTRODUCTION}

Liquid propulsion rocket engines employ ducts to route propellant and oxidizer among the various turbomachinery components. Duct design, including cross sectional shape, area, and amount of bending as a function of axial distance, influences the presence and extent of secondary flows and flow separation near the wall. These phenomena determine the pressure losses generated in the duct. Secondary flows also influence the velocity distribution and dynamic loads experienced by downstream components. The present study was performed in order to identify a duct geometry that reduces the dynamic loads experienced by a LOX turbopump located downstream of a 90 deg elbow.

This paper presents the results of a series of numerical simulations for several design geometries of a 90 degree elbow, a common feature in radial-engine turbomachinery. Prior to presenting the LOX duct simulations, results are presented for a benchmark simulation (water flow through a 90 degree elbow) to demonstrate the applicability of compressible flow codes to an incompressible flow. Results are then presented for several duct geometries, including constant as well as varying cross sectional shape and area.

\section{NUMERICAL PROCEDURES}

Ducts do not generally fall into a single class of engine components, e.g., ducts are found both in turbomachinery and combustion devices. Therefore, the three principal codes used to analyze turbomachinery and combustion devices at MSFC were applied in the current study. This also presented the unique opportunity to cross check the codes.

The governing equations in the computational fluid dynamics (CFD) analysis are the time-dependent, three-dimensional Reynolds-averaged Navier-Stokes equations. Three CFD algorithms were used during this study. The first algorithm is Corsair, a densitybased, time marching, implicit, finite-difference

\footnotetext{
\# Aerospace Engineer, Member AIAA; ‘ Aerospace Engineer, Associate Fellow AIAA; + Computer Scientist. Copyright $\bigcirc 2003$ by the American Institute of Aeronautics and Astronautics, Inc. No copyright is asserted in the United States under title 17, U.S. Code. The U.S. Government has a royalty-free license to exercise all rights under the copyright claimed herein for Government Purposes. All other rights are reserved for the copyright owner.
} 
scheme. Corsair was developed for studying turbomachinery components. The procedure is thirdorder spatially accurate and second-order temporally accurate. The inviscid fluxes are discretized according to the scheme developed by Roe [1]. The viscous fluxes are calculated using standard central differences. An approximate-factorization technique is used to compute the time rate changes in the primary variables. Newton sub-iterations are used at each global time step to increase stability and reduce linearization errors. For all cases investigated in this study, one Newton sub-iteration was performed at each time step. To extend the equations of motion to turbulent flows, an eddy viscosity formulation is used. The turbulent viscosity is calculated using the twolayer Baldwin-Lomax algebraic turbulence model [2]. Algebraically generated $\mathrm{H}$-grids are used to discretize the remainder of the flow field. The code has been parallelized using Message Passing Interface (MPI) and OpenMP application program interfaces (API's) to reduce the computation time for large-scale threedimensional simulations.

The second and third algorithms were developed for analyzing combustion devices. The second CFD algorithm used for this study is FDNS, a pressurebased, finite-difference Navier-Stokes solver. The algorithm is second-order accurate and uses the extended $\mathrm{k}$-e turbulence model and wall functions. The three-dimensional, steady, incompressible version was used with a structured grid.

The third CFD algorithm used for this study is Chem, which is a density-based, finite-volume Navier-Stokes solver. The algorithm is implemented using the Loci framework, which allows implementation issues such as parallel processing to be handled transparently to the coding of the CFD algorithm [3]. The turbulent viscosity is calculated using the decoupled version of the baseline two-equation turbulence model [4]. Three-dimensional, structured grids were created using a highly-automated grid generator. Since Chem is strictly a perfect gas code, a method was introduced to approximate liquids. A large gauge pressure was chosen such that normalized density fluctuations were small. The molecular weight was chosen to match the density of the liquid at gauge pressure, and the transport properties of the liquid were specified.

\section{BOUNDARY CONDITIONS}

For incompressible inlet flow the mass flow, total temperature, and the circumferential and radial flow angles are specified as a function of the radius. The static pressure is extrapolated from the interior of the computational domain.

For incompressible outflow the circumferential and radial flow angles, total pressure, and the total temperature are extrapolated from the interior of the computational domain. The static pressure is specified at mid-span of the computational exit and the pressure at all other radial locations at the exit is obtained by integrating the equation for radial equilibrium. Periodicity is enforced along the outer boundaries of the $\mathrm{H}$-grids in the circumferential direction.

At solid surfaces the velocity is set to zero, the normal derivative of the pressure is set to zero, and the surfaces are assumed to be adiabatic.

\section{BENCHMARK TEST CASE}

To confirm the applicability of Corsair and Chem to incompressible flows, two benchmark test cases were performed for the flow of water through a 90-degree elbow [5]. Laser Doppler Velocimetry (LDV) and static pressure data were obtained as part of the experiments. The experiment was performed for a Reynolds number of 43,000 and a mass flow 3.6 $\mathrm{lbm} / \mathrm{sec}$.

The Corsair and Chem codes produced similar results, therefore the results from the Corsair code only are presented for brevity. Two views of the Corsair computational grid are shown in Fig. 1. The grid contains 385 points in the streamwise direction, 93 points in the circumferential direction and 41 points in the radial direction. Figure 2 shows a comparison of the predicted and experimental velocity contours 1.0 duct diameter downstream of the elbow. The predicted and experimental results exhibit good agreement. Figure 3 shows a comparison of experiment and computed horizontal and vertical mean streamwise velocity $U / U_{B}$ in the turbulent flow $\sim 0.6$ diameters upstream of the bend. Prominent features include inlet boundary layers of depth $\sim 0.09 \mathrm{~d}$ and large central region of uniform velocity. Figure 4 shows a comparison of the predicted and experimental wall static pressure distributions in the region of the elbow. Good agreement exists between the predicted and experimental results, suggesting that the Corsair compressible flow code is suitable for certain incompressible flow simulations. Results for the benchmark case have been reproduced using much coarser grids, e.g., 94 percent fewer grid points. 


\section{LOX PUMP INLET DUCT CASES}

Two duct cases were simulated using Corsair and FDNS. Figure 5 shows two computational grids (Elbow \#1 and Elbow \#2) used to represent a $90 \mathrm{deg}$ elbow at the inlet to a LOX pump. Elbow \#1, simulated using Corsair, has a constant, circular cross section in the axial direction. The inlet of Elbow \#2, simulated using FDNS, transitions to a triangular cross section at midsection of the bend with the apex along the outer wall. The triangular cross section transitions to a circular cross section with 15 percent greater area. Each computational grid contains $191 \times 93 \times 41$ points in the streamwise, circumferential, and radial directions, respectively. Sufficient grid spacing was chosen near the wall to resolve the boundary layer. Figure 6 shows the computed exit velocities for Elbow \#1 (left) and Elbow \#2 (right); vectors are aligned in the radial-circumferential plane. A secondary flow is clearly evident in both cases, similar to the flow distribution first reported by Prantdl [6]. The circulation is symmetric in Elbow \#1, but asymmetric in Elbow \#2. Figure 7 shows the predicted velocities for Elbow \#1 and Elbow \#2 (with vectors in axial-radial plane). The expansion of the flow path near the exit of Elbow \#2 causes it to act as a diffuser. Hence, the flow encounters an adverse pressure gradient, resulting in separation. Pressure losses are considerably less for the Elbow \#1 design.

\section{PARAMETRIC STUDIES}

Results from the LOX inlet duct case studies showed that duct cross-sectional shape and area influence flow conditions at the exit and, therefore, inducer performance. A series of parametric studies was therefore undertaken to study the respective influences of pipe bend radius, cross sectional shape, and area. Figure 8 illustrates the geometry parameters. The Chem algorithm was used with structured threedimensional grids. Circular, constant-area, straight cross-sections of length 1-diameter and 2-diameter were added at the elbow inlet and exit, respectively. Water was chosen as the working fluid for consistency with the benchmark results. All cases were run at a turbulent Reynolds number of 43,000 . A gauge pressure of 100 psia was chosen which resulted in a molecular weight of $3523 \mathrm{~kg} / \mathrm{kmol}$. For the following results, normalized density fluctuations were indeed small, approximately 0.2 percent. Figure 9 shows an example grid for bend radius, pipe radius, and cross sectional shape of $10.0 \mathrm{in,} 3 \mathrm{in}$, and circular, respectively.
Flow conditions in a bent duct may be described as the result of a balance between centrifugal and pressure gradient forces, expressed as

$$
\frac{\Delta P}{d}=\rho \frac{\bar{U}^{2}}{R}
$$

where $\Delta \mathrm{P}=\left(\mathrm{P}_{\text {outer }}-\mathrm{P}_{\text {inner }}\right)$ is the static pressure difference between inner and outer walls of the bend. Results for the benchmark case show good agreement with this relationship (Fig. 4).

\section{Pressure Loss}

A pressure loss coefficient may be defined as the difference in total pressure between inlet and exit of the duct, normalized by inlet dynamic pressure:

$$
\frac{d P_{\text {total }}}{q_{\text {in }}}=\frac{P_{\text {inlet }}-P_{\text {exit }}}{\frac{1}{2} \rho V^{2}}
$$

Figure 10 summarizes pressure loss coefficients as a function of duct bend radius and duct diameter for circular cross sections; the larger the symbol, the greater the loss. Several subsets of case studies are summarized here. In the first, pipe radius was fixed at 1.0 in and bend radius was varied as represented by the vertical distribution of symbols. Minimal pressure losses occur for the shortest bend radii, since boundary layer growth (hence blockage) is proportional to distance from the inlet. As expected, the strength of the secondary flow is inversely proportional to the bend radius. In the second subset of cases, the ratio between bend radius and pipe radius was fixed at 10:1, represented by the diagonal distribution of symbols. Losses are relative constant at small pipe radius, but decrease significantly for larger radius. This behavior is due to two reasons. First, blockage by boundary layer growth (for larger bend radius) is offset by larger duct diameter. Second, the secondary flow is also weaker at larger bend radius, hence less blockage. In the third subset, the bend radius was fixed at 10.0 in and duct radius was varied, represented by the horizontal distribution of symbols. For smaller radii, the trend is generally toward decreasing pressure loss with increasing duct radius.

\section{Flow Angle Variation}

To relate the water-based CFD results to those of the two LOX duct cases, the pump affinity law was used,

$$
\frac{Q_{L O X}}{N_{L O X}}=\frac{Q_{H 2 O}}{N_{H 2 O}}
$$


where $\mathrm{Q}$ and $\mathrm{N}$ refer to volume flow rate and wheel speed, respectively. The absolute flow angle, one pipe diameter downstream of the elbow exit, may be defined as

$$
\phi=\tan ^{-1}\left(\frac{V_{r}}{V_{f}}\right)
$$

where $V_{r}$ is the tangential speed of a hypothetical pump blade at a given radius, $r$, and $V_{f}$ is the magnitude of the streamwise flow velocity. Figure 11 illustrates absolute flow angle for the case of a bend radius and pipe radius of 10 in and 3 in, respectively. Figure 12 shows the azimuthal variation in absolute flow angle at 10,50, and 90 percent of pipe radius. Maximum angular differences are $0.37,10.94$, and $11.57 \mathrm{deg}$, respectively. The corresponding incidence angle (i.e., angle at which the flow meets the blade) is not constant, except perhaps near the hub of the pump. Obviously, this behavior cannot be eliminated simply by manufacturing the pump with a different, radially-dependent inlet blade angle.

Figure 13 summarizes maximum difference in flow angle at 90 percent span for all of the parametric cases shown in Fig. 10; the larger the symbol, the greater the angular difference. For constant pipe radius, the angular difference is inversely proportional to the bend radius. This is consistent with the strength of the circulation being proportional to the pressure difference between inner and outer bend. For constant bend radius, the angular difference is inversely proportional to pipe radius.

\section{DISCUSSION}

Comparison of Figs. 10 and 13 shows that an optimal duct design must balance the pressure loss against the angular difference, respectively, arising from the presence of the secondary flow. Ducts with larger bend radius are undesirable owing to large pressure loss. Moreover, the largest one or two bend radii considered in the present study may not be feasible to implement for practical reasons. Ducts with smaller pipe radius will generally experience larger flow angular difference and larger pressure loss. Therefore, the optimal duct design with $90 \mathrm{deg}$ bend should favor relatively larger bend radius and larger pipe radius.

\section{CONCLUSIONS}

A series of three-dimensional Navier-Stokes simulations has been performed to identify an optimum design for a $90 \mathrm{deg}$ pipe bend at the inlet of a hypothetical LOX pump. The presence of a secondary flow may increase high-cycle fatigue, cause flow separation near the pump inlet, cause additional flow blockage in the blade passages, and lower overall pump efficiency. Large pipe radius is preferred to minimize the strength of the secondary flow, which leads to variations in flow angle.

\section{ACKNOWLEDGEMENTS}

The authors would like to acknowledge the use of the supercomputer facilities at NASA Ames Research Center, and especially thank Mr. Chuck Niggley for his assistance.

\section{REFERENCES}

1. Roe, P. L., "Approximate Riemann Solvers, Parameter Vectors, and Difference Schemes," Journal of Computational Physics, Vol. 43, 1981, pp. 357-372.

2. Baldwin, B. S., and Lomax, H., "Thin Layer Approximation and Algebraic Model for Separated Turbulent Flow," AIAA Paper 78-257, Huntsville, AL, January, 1978.

3. Luke, E. , "Loci: A Deductive Framework for Graph-Based Algorithms," Third International Symposium on Computing in Object-Oriented Parallel Environments, San Francisco, CA, USA, December 1999, Springer-Verlag, pp. 142-153.

4. Menter, F. R., "Two-equation Eddy-viscosity Turbulence Models for Engineering Applications," AIAA Journal, Vol. 32, No. 8, August, 1994.

5. Enayet, M. M., Gibson, M. M., Taylor, A. M. K. P. and Yianneskis, M., "Laser-Doppler Measurements of Laminar and Turbulent Flow in a Pipe Bend," Int. J. Heat \& Fluid Flow, 1982, Vol. 3, pp. 213-219.

6. Prandtl, L., "Neuere Ergebnisse der Turbulenzforschung. Z. VDI, 1933, Vol. 77, pp. 105-114. 

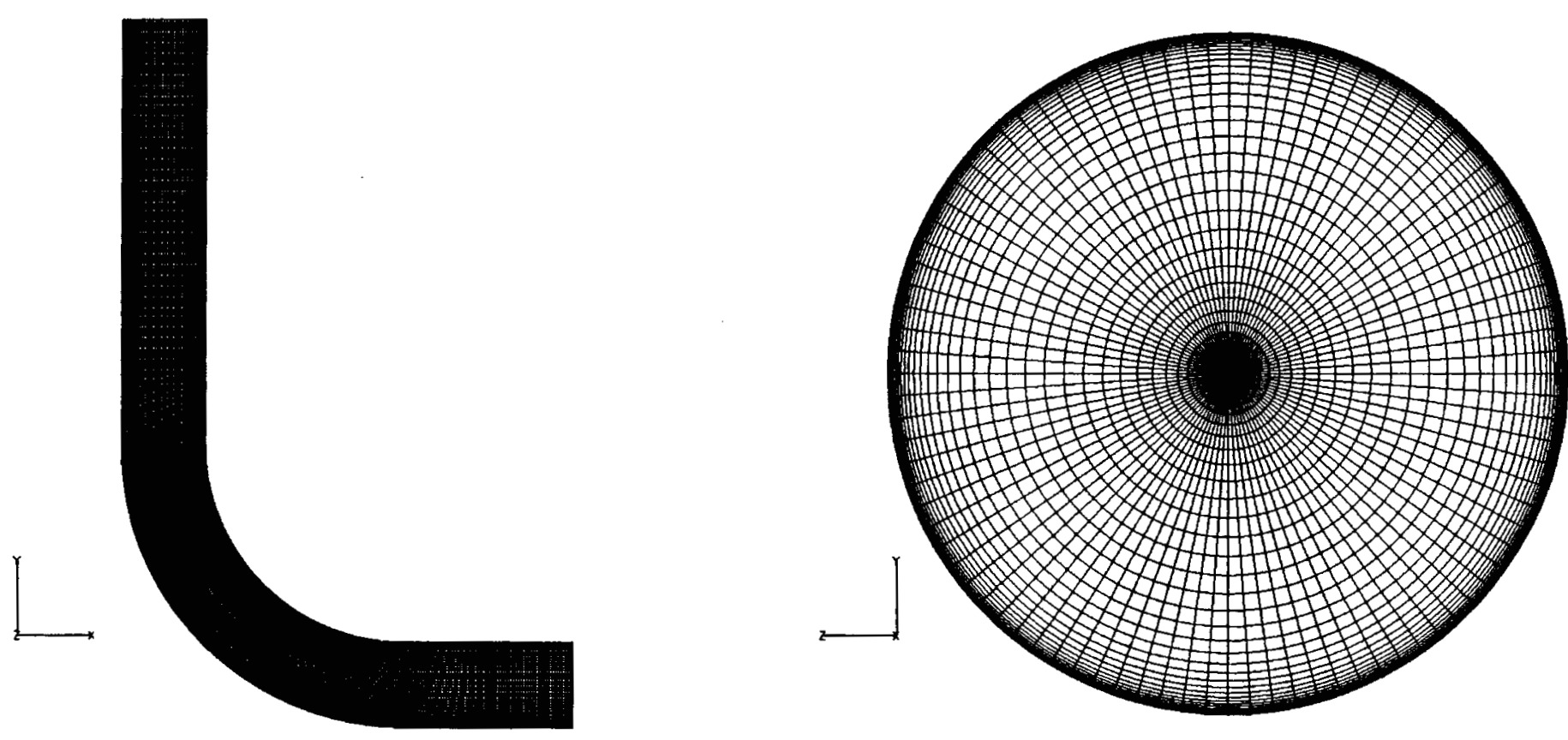

Figure 1. Two views of the computational grid for the benchmark pipe bend case. 
CFD

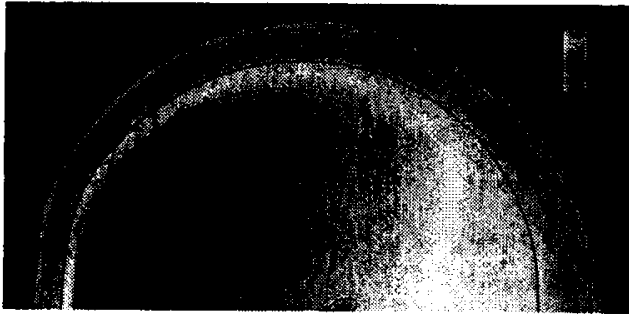

Expt

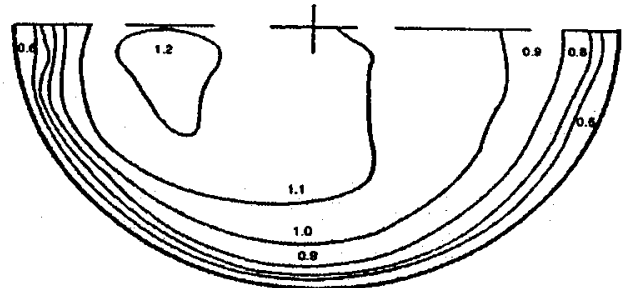

30 deg of curve

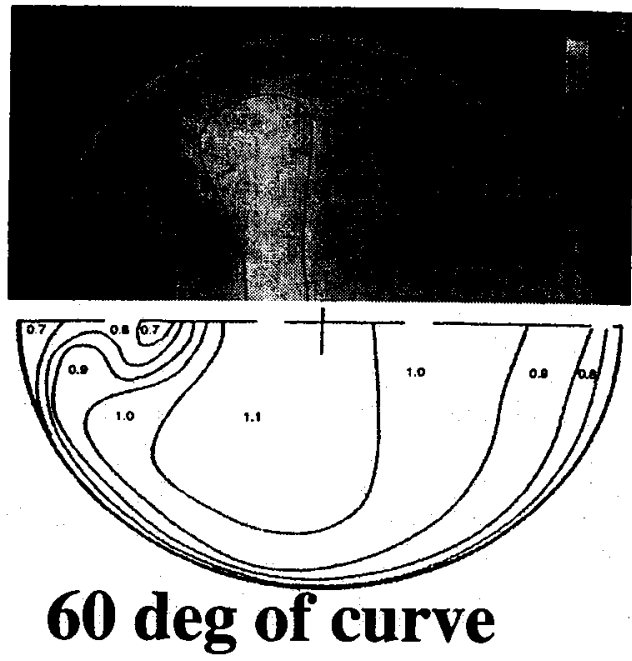

CFD

Expt
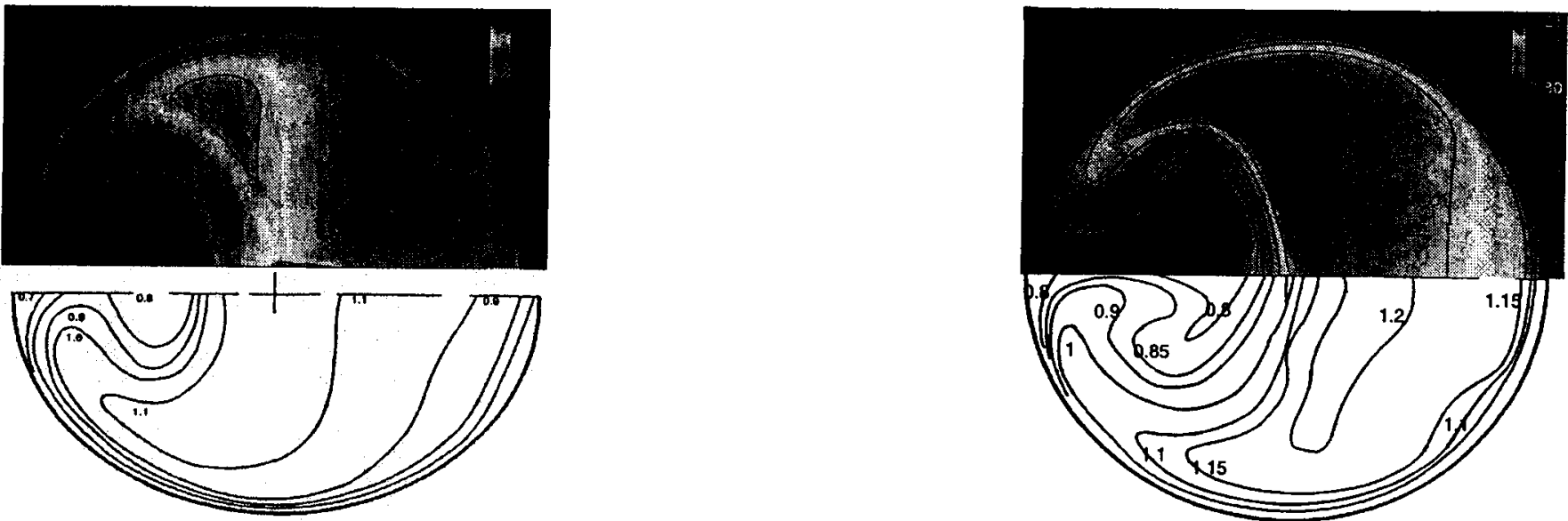

75 deg of curve

\section{1-dia downstream}

Figure 2. Comparison of predicted and experimental non-dimensional velocity contours (upper left to lower right) at 30,60, and $75 \mathrm{deg}$ of curve, and 1 duct diameter downstream of the elbow. 


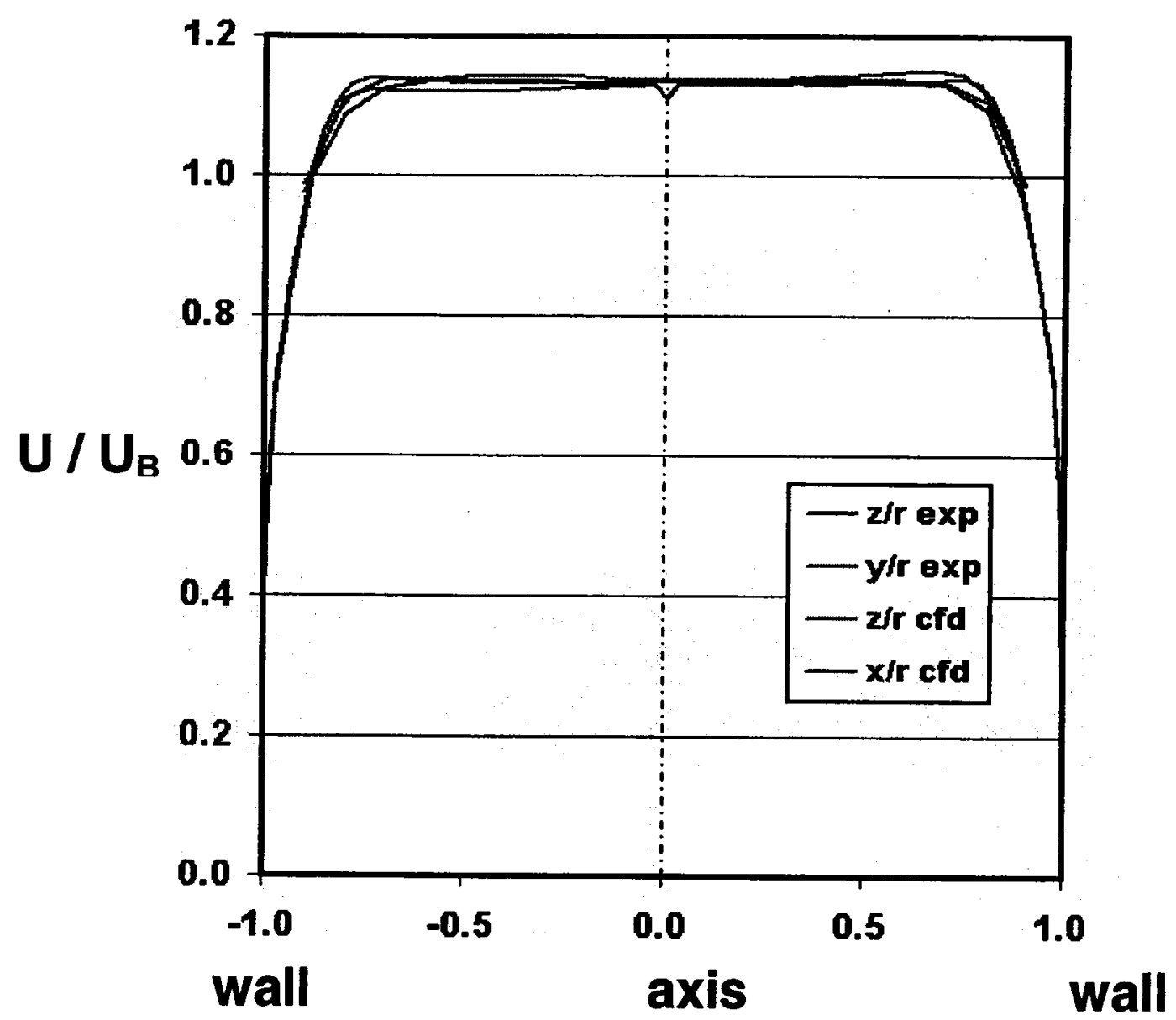

Figure 3. Profiles of streamwise nondimensional velocity $U / U_{B}$ in the turbulent approach flow 0.58 diameter upstream of the bend.

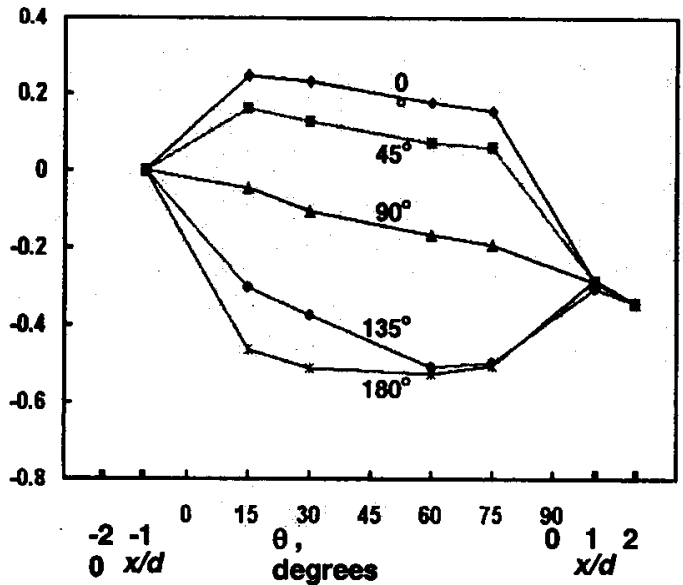

CFD

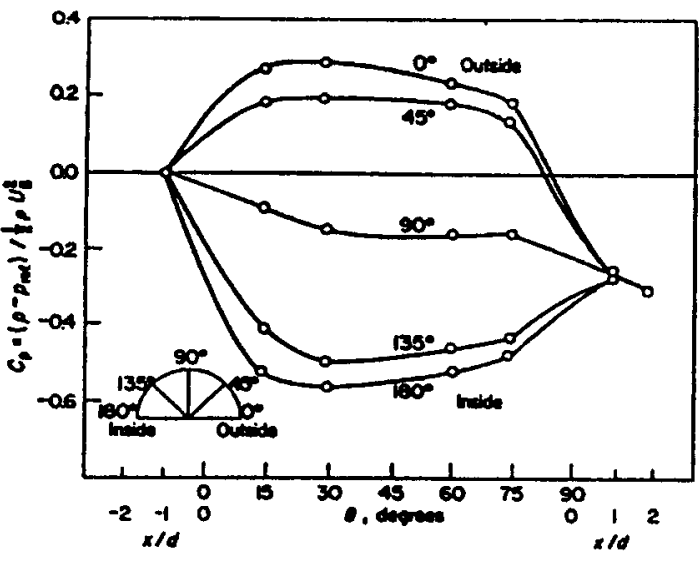

Experiment

Figure 4. Predicted (left) and experimental (right) wall static pressure distributions in the region of the elbow. 


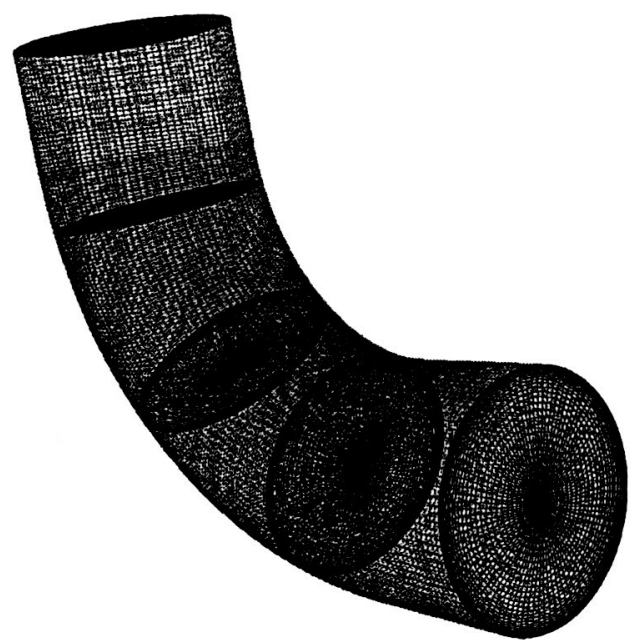

Elbow

\#1

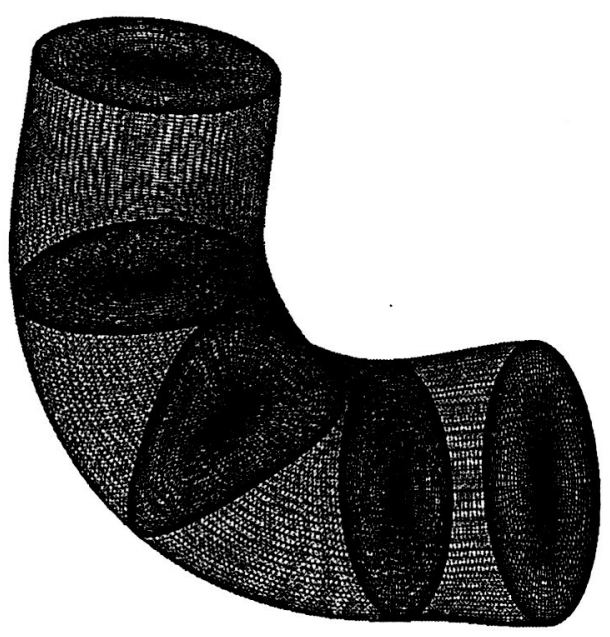

Elbow

\#2

Figure 5. Computational grids for $90 \mathrm{deg}$ elbow cases employing circular (left) and triangular cross section. Inlet is from above.

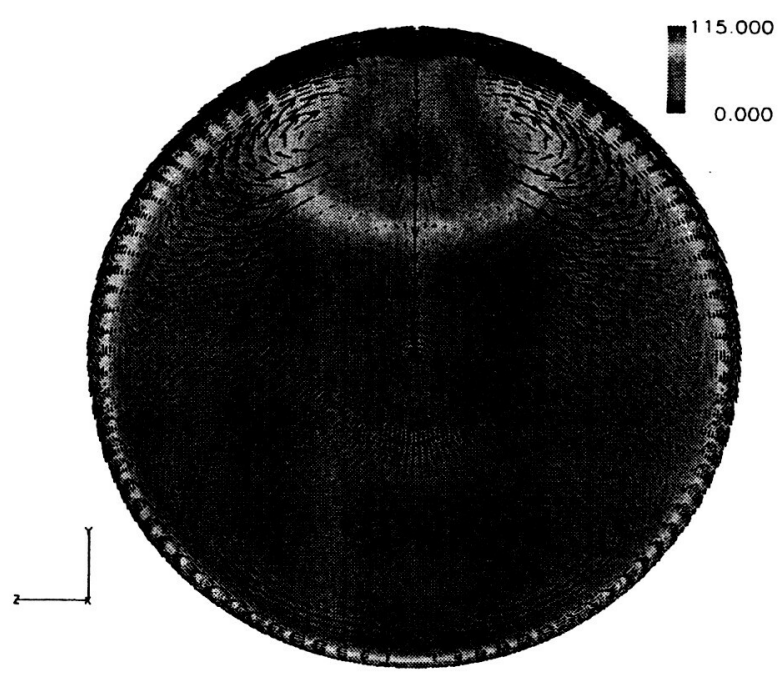

Elbow \#1

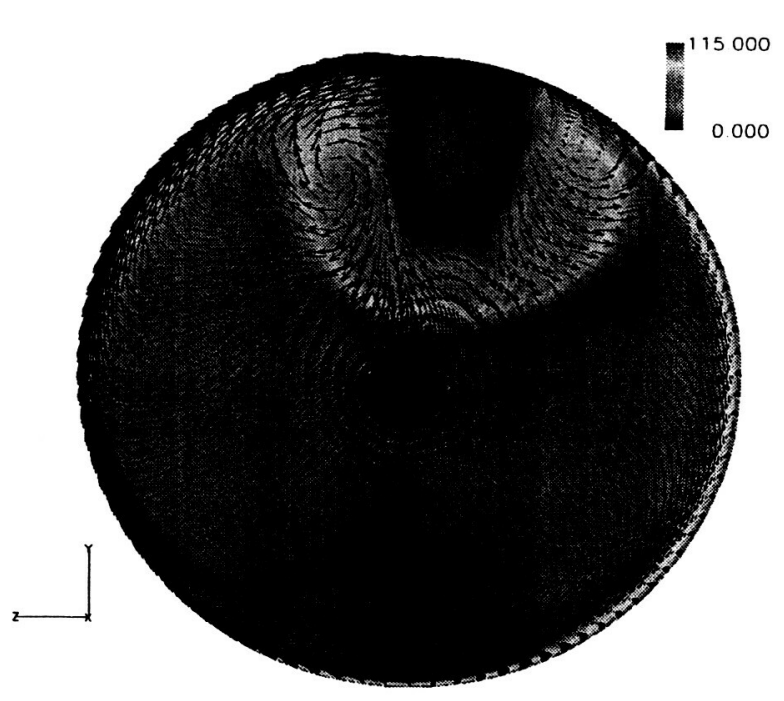

Elbow \#2

Figure 6. Comparison of predicted exit velocities for Elbow \#1 (left) and Elbow \#2 (right); vectors are in radial-circumferential plane. 


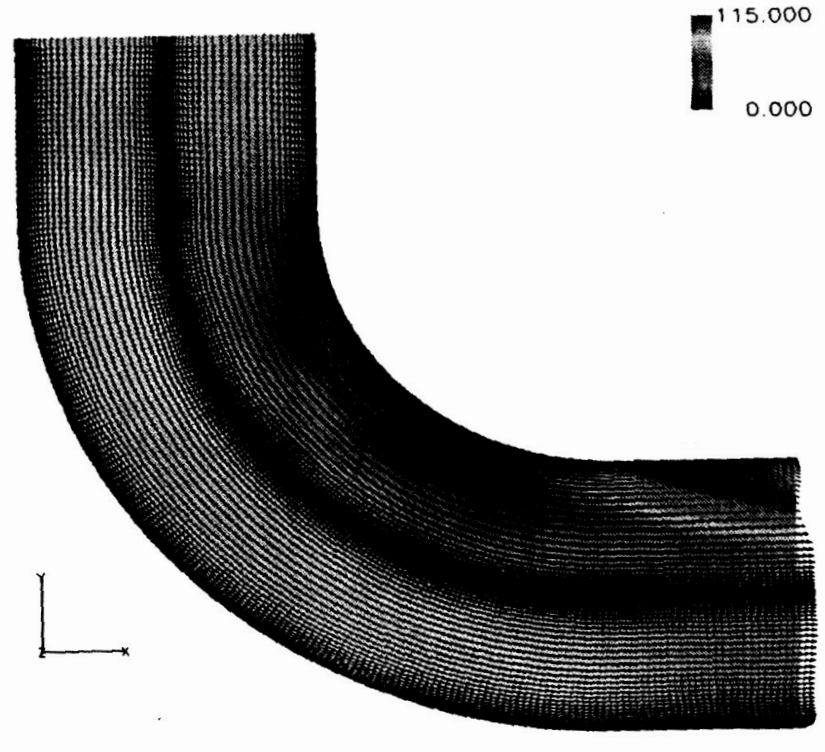

Elbow \#1

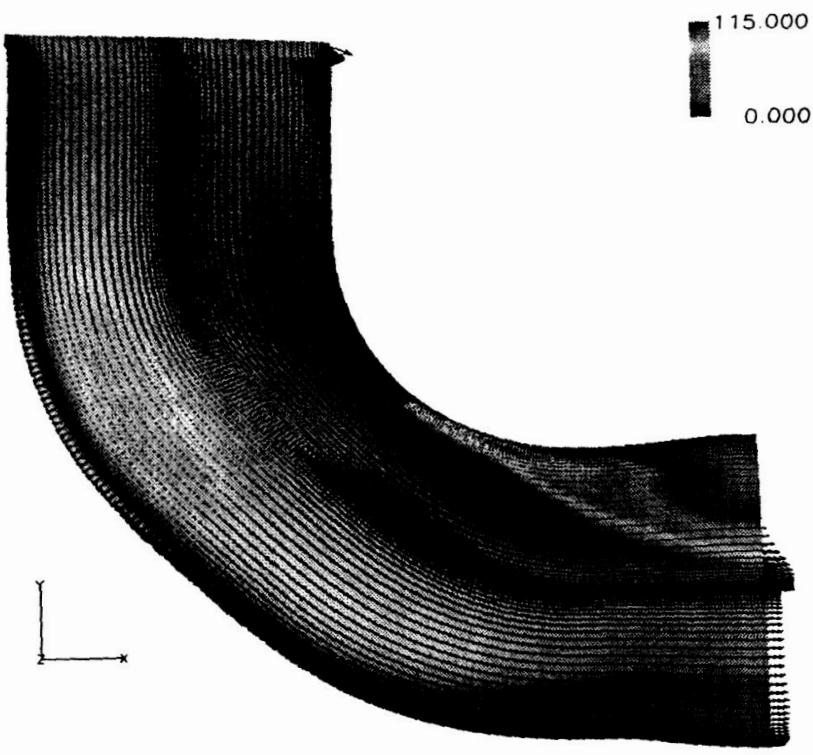

Elbow \#2

Figure 7. Comparison of predicted exit velocities for Elbow \#1 (left) and Elbow \#2 (right); vectors are in axial-radial plane. Expansion of cross section near the exit of Elbow \#2 induces flow separation. 


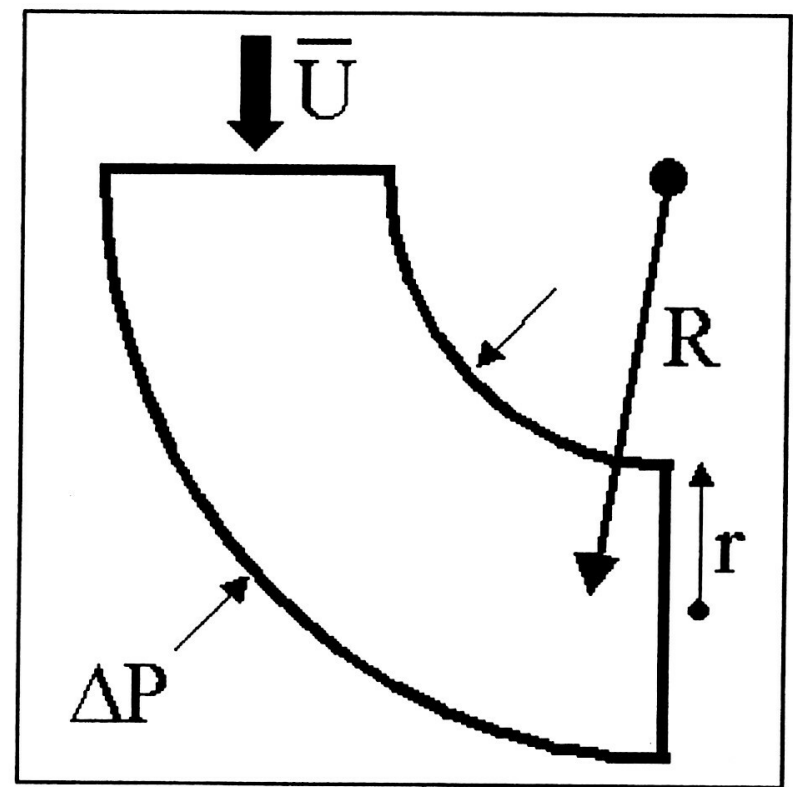

Figure 8. Geometric parameters for three-dimensional pipe parametric study.
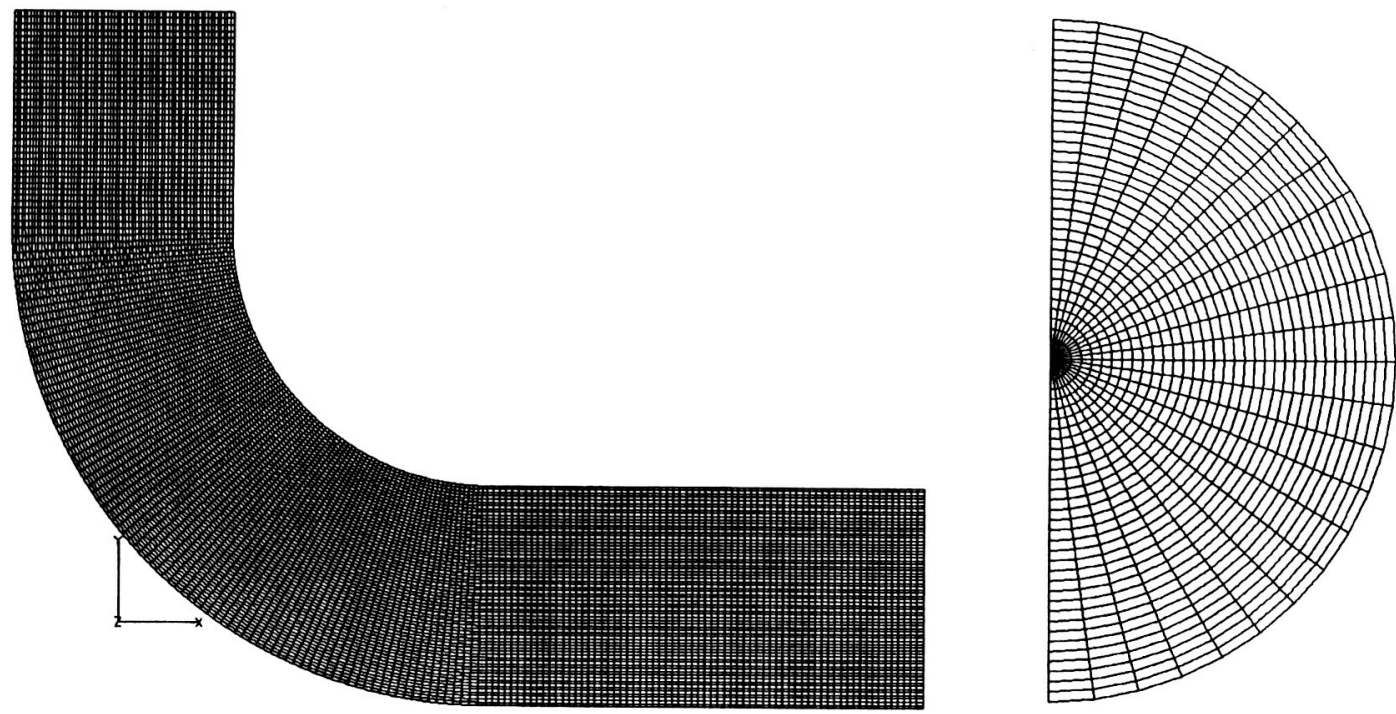

Figure 9. Two views of a grid used for the pipe parametric study (in this example, bend radius and pipe radius of 10 in and 3 in, respectively). 


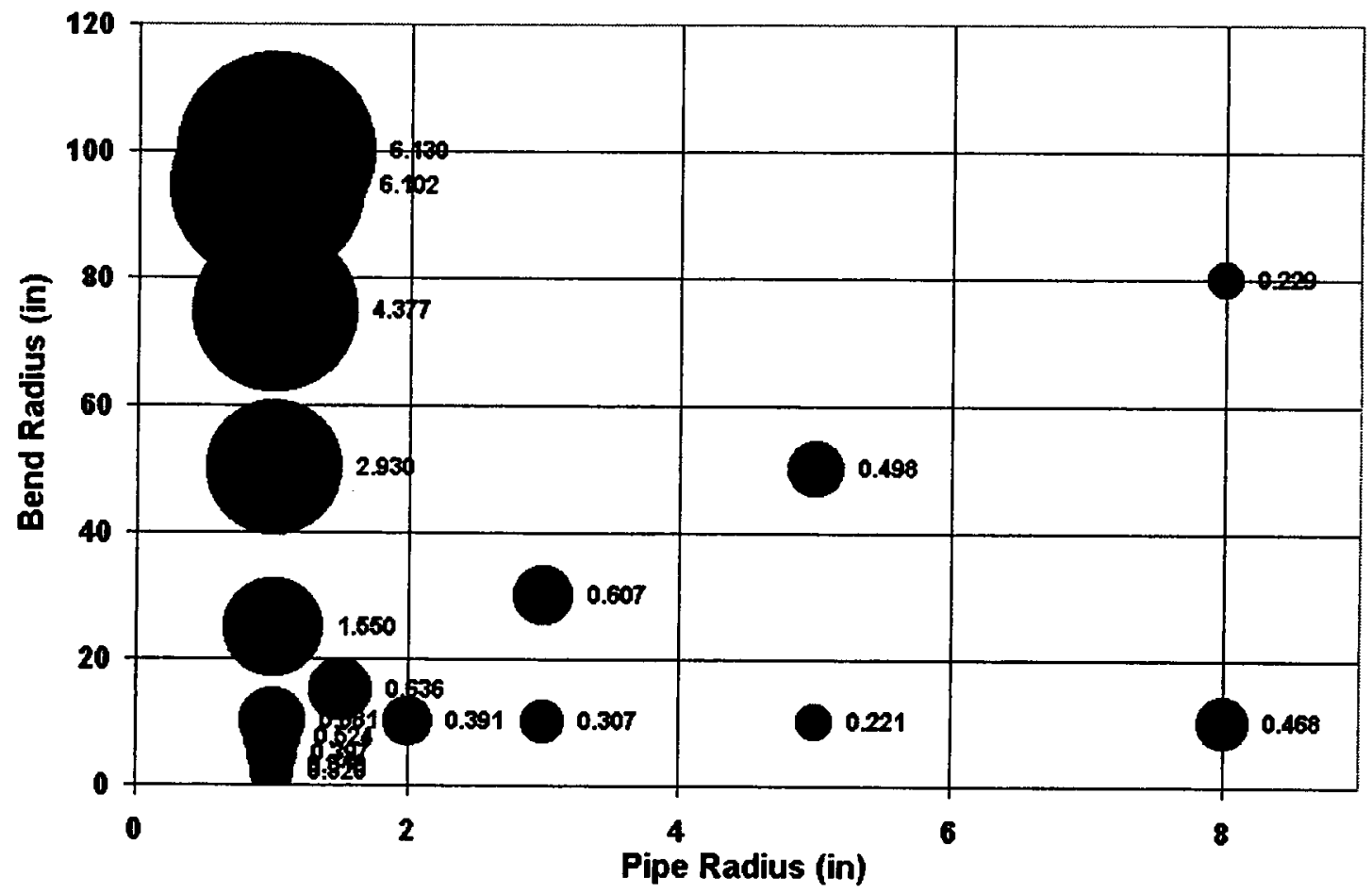

Figure 10. Map of pressure loss coefficients for ducts with circular cross section. A larger symbol corresponds to greater losses, hence a less-desirable feature. 


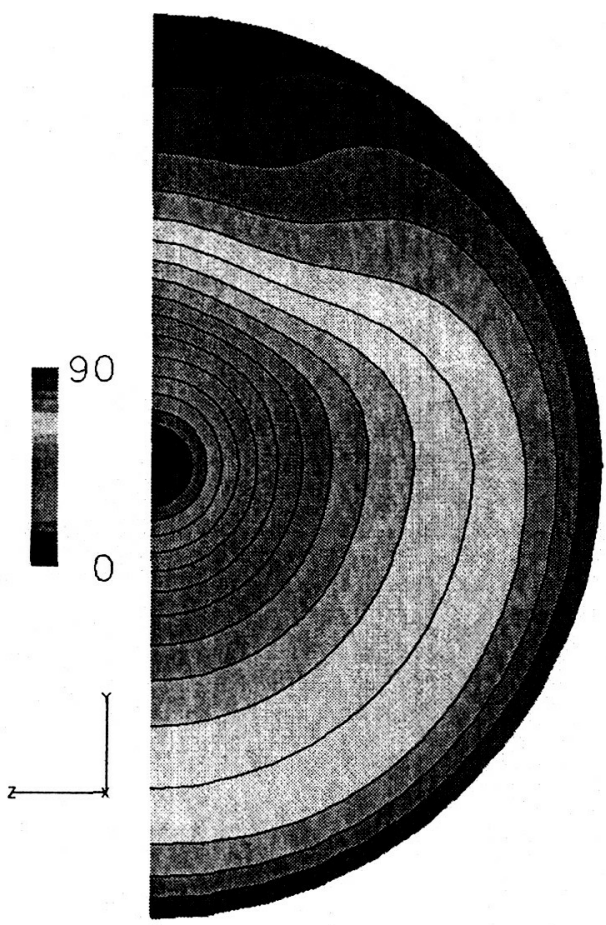

Figure 11. Example distribution of absolute flow angle at one diameter downstream from 90 deg pipe bend.

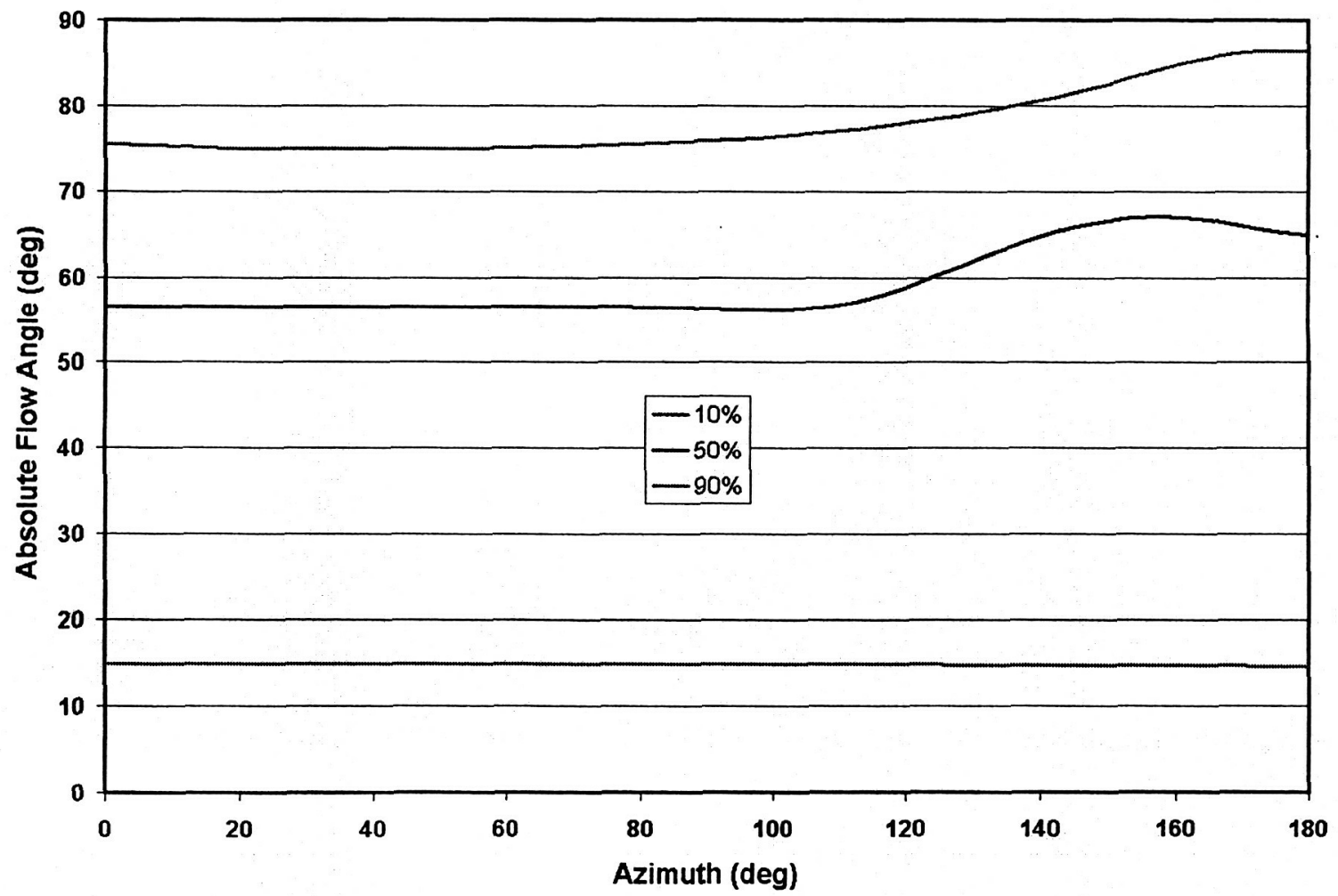

Figure 12. Absolute flow angle as a function of circumferential angle ( $0 \mathrm{deg}$, inside) at 10,50 , and 90 percent of pipe radius, based on Fig. 11. 


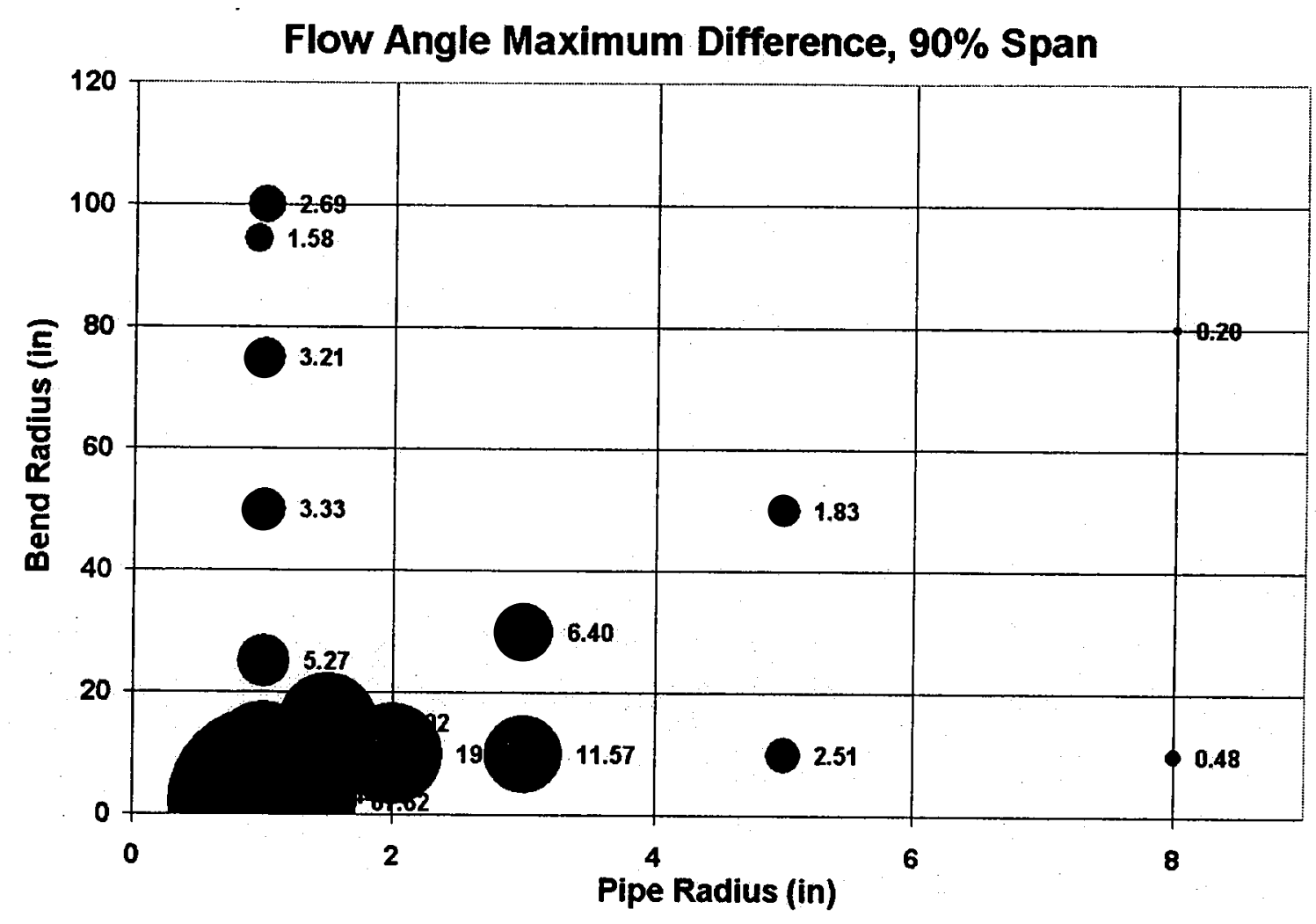

Figure 13. Maximum flow angle variation for CFD cases. A larger symbol corresponds to greater angular variation, hence a less-desirable feature. 